\title{
Modeling the large inelastic deformation response of non-filled and silica filled SL5170 cured resin
}

\author{
Z. WU \\ Department of Mechanical Engineering and Applied Mechanics, University of Michigan, Ann \\ Arbor, Michigan, 48109, USA \\ S. AHZI* \\ Université Louis Pasteur,IMFS UMR7507 CNRS-ULP, 2 Rue Boussingault, 67000, \\ Strasbourg, France \\ E-mail: Said.Ahzi@ipst-ulp.u-strasbg.fr \\ E. M. ARRUDA \\ Department of Mechanical Engineering and Applied Mechanics, University of Michigan, Ann \\ Arbor, Michigan, 48109, USA
}

\author{
A. MAKRADI \\ Center for Advanced Engineering Fibers and Films,Clemson University, SC 29634, USA
}

\begin{abstract}
In recent years, important efforts have been focused on rapid production of tools using Rapid Prototyping and Manufacturing (RP\&M) technologies such as the Stereo-Lithography Apparatus (SLA). One of the applications is the development of rapid polymer tooling such as dies for injection molding. For these applications, optimal thermal as well as mechanical properties of final tools are of significance. In order to characterize the mechanical response of materials made by SLA, a standard set of material tests, including uniaxial tension and compression tests under different strain rates and different temperatures, was conducted for both silica filled and non-filled resin. In this paper, the mechanical response of the non-filled SL5170 cured resin is discussed in terms of an elastic-viscoplastic material model. Further, a new model for silica filled SL5170 cured resin was developed to estimate the stress-strain relationship of the composite. This composite model is an extension of the elastic-viscoplastic model for non-filled resin to include the elastic deformation of the silica particles. The stress-strain curves predicted by the models under homogeneous deformation show good agreement with the experimental results.

(c) 2005 Springer Science + Business Media, Inc.
\end{abstract}

\section{Introduction}

Of all revolutionary Rapid Prototyping and Manufacturing (RPM) technologies, the Stereo-Lithography Apparatus (SLA) is one of the first commercially available layer-additive process to enable the rapid generation of physical objects directly from a computer-aided design (CAD) database. Recently, in SLA technology new materials have been explored to enhance the mechanical properties of the prototypes, so that the improved rapid parts can be directly used for manufacture.

SL5170 is an attractive resin for the development of rapid polymer tools. Compared to the earlier SLA acrylate photopolymer systems, parts built using the photopolymer SL5170 have significantly enhanced mechanical properties, that is, a high level of overall accuracy, negligible curl, improved flatness, minimal "green creep" distortions, and better tensile and flexural moduli [1]. The polymer tools for injection molding can be made from non-filled pure or filled resins. Silica filled SL5170 has previously been examined for the fabrication of a polymer composite die. ASTM D638 Type I dog-bone cavity dies were built from unfilled and silica filled resin. After injection of polyester parts, the composite material was found to have higher resistance as compared to the pure resin [2].

SLA produces solid parts by means of a chemical reaction known as photo-polymerization of the resin. Post-cured SL5170 parts have a high degree of polymerization and cross-link density. An investigation of the extent of SL5170 polymerization found a usable energy exposure per layer of 1000 to $2000 \mathrm{~J} / \mathrm{m}^{2}$ was required to achieve a suitable cure depth and sufficient mechanical strength for this material. The extent of

\footnotetext{
* Author to whom all correspondence should be addressed.

${ }^{\dagger}$ Present Address: Institut Supérieure de la conception, ERMeP, 27 rue d'Hellieule, 88100, St-Die-des-Vosges, France. 
polymerization for this case would be about $85 \%$. In addition, postcuring is conducted in practice to reach a higher degree of polymerization [3]. Cross-linking involves formation of strong covalent bonds that connect the polymer chains. Since the chemical forces must be overcome before molecular flow can be achieved, the solidified SL5170 part cannot melt upon heating [1]. Therefore, SL5170 cured resin is classified as a thermoset polymer. It has a glass transition temperature of between 60 and $80^{\circ} \mathrm{C}$. Below this temperature SL5170 is glassy and above it the resin is rubbery

\subsection{Review of material models for rubbery and glassy polymers}

Various models have been developed to explain the mechanical response of rubbery and glassy polymers. Treloar [4] reviewed physically based models for rubber-elasticity and developed a Gaussian network theory for rubbers. In his theory, the entropy of the network and the difference in the entropy of deformation, are calculated by relating the change in molecular chain dimensions to the macroscopic strain, as illustrated by the following equation

$$
\Delta S_{\mathrm{E}}=-\frac{1}{2} n k\left(\lambda_{1}^{2}+\lambda_{2}^{2}+\lambda_{3}^{2}-3\right)
$$

where $\Delta S_{\mathrm{E}}$ is the change in entropy on deformation, $n$ is the chain density, $k$ is Boltzmann's constant and $\lambda_{1}$, $\lambda_{2}$, and $\lambda_{3}$, are the three principal stretches. Equation 1 is based on affine deformation of network cross-links and incompressibility assumptions. It is valid for small strains where the stretches applied do not approach the limiting extensibility of the polymer chains.

To overcome the limitation of the Gaussian formula of Equation 1 the inverse Langevin function was introduced in the non-Gaussian uniaxial force-extension relation by James and Guth [5]:

$$
\begin{aligned}
f= & \frac{1}{3} n k \theta N^{\frac{1}{2}} \\
& \times\left[L^{-1}\left(\lambda N^{-\frac{1}{2}}\right)-\lambda^{-\frac{3}{2}} L^{-1}\left(\lambda^{-\frac{1}{2}} N^{-\frac{1}{2}}\right)\right],
\end{aligned}
$$

where $f$ is the force on the network, $\lambda$ is the extension ratio, $\theta$ is the absolute temperature, and $\sqrt{N}$ is the limiting extensibility of the network, i.e., the locking stretch. The Langevin function is defined by

$$
L(y)=\operatorname{coth}(y)-\frac{1}{y}=x
$$

with the inverse form given as

$$
L^{-1}(x)=y \text {. }
$$

An amorphous polymer is considered glassy at temperatures below its glass transition temperature $\theta_{g}$, and rubbery at temperatures above its $\theta_{g}$. In the glassy regime, the initially isotropic solid exhibits a linear elastic response followed by yielding. The initial yielding of the material is known to depend on pressure, strain rate, and temperature. After yielding, the material may exhibit a strain softening. As larger strains are approached, the material strain hardens.

It has been previously documented that a glassy polymer must overcome two physically distinct sources of resistance before large inelastic flow may occur [6]. Argon [6] derived a model for local shear transformations in glassy polymers based on a double-kink model of a chain segment rotating against the elastic impedance of surrounding chains as one barrier to inelastic flow. Boyce et al. [7] extended Argon's model and proposed a three-dimensional constitutive model for large elasticviscoplastic deformation of glassy polymers, which incorporated yield and post-yield behavior of glassy polymers including pressure dependence, rate and temperature dependence, and true strain softening. Yielding and the accumulation of inelastic strain is expressed by

$$
\dot{\gamma}^{\mathrm{p}}=\dot{\gamma}_{0} \exp \left[-\frac{A S_{0}}{\theta}\left(1-\left(\frac{\tau}{S_{0}}\right)^{\frac{5}{6}}\right)\right]
$$

or by,

$$
\tau=S_{0}\left[1-\frac{\theta}{A S_{0}} \ln \left(\frac{\dot{\gamma}_{0}}{\dot{\gamma}^{\mathrm{p}}}\right)\right]^{\frac{6}{5}}
$$

where

$$
A \equiv \frac{39 \pi \omega^{2} \alpha^{3}}{16 k}
$$

Here $A$ is the activation volume, $\omega$ is net angle of rotation of the molecular segment, and $\alpha$ is the mean molecular radius and $S_{0}$ is approximated given by.

$$
S_{0} \equiv \frac{0.076 \mu}{1-v}
$$

$S_{0}$ is termed the athermal shear yield strength of the material because $\tau$ approaches $S_{0}$ when the temperature approaches absolute zero provided that the plastic strain rate is finite. $\dot{\gamma}^{\mathrm{p}}$ is the plastic strain rate, $\dot{\gamma}_{\mathrm{o}}$ is a pre-exponential factor, $\mu$ is the shear modulus and $\nu$ is Poisson's ratio. According to Equation 5, the yield strength $\tau$ is dependent on the plastic strain rate and temperature.

In the constitutive model of Boyce et al. [7], strain softening is incorporated into the constitutive law by evolving the athermal yield strength, $S_{0}$, of the material when plastic straining starts. The evolution of the shear resistance is thus defined as

$$
\dot{S}=h\left(1-\frac{S}{S_{\mathrm{ss}}}\right) \dot{\gamma}^{\mathrm{p}}
$$

where $S$ is the current athermal deformation resistance of the material indicating the current state of structure, $h$ is the slope of the yield drop with respect to plastic strain, $S_{\mathrm{ss}}$ is the value of $S$ at steady state, i.e., the preferred structure determined by temperature and 
strain rate, $\dot{S}$ and is the softening evolution of the shear resistance.

The peak shear yield strength of amorphous polymers has been found to be essentially linearly dependent upon pressure for moderately large hydrostatic stresses. Boyce et al. [7] combined this with the athermal shear resistance $S$

$$
\bar{S}=S\left(1+\alpha_{\mathrm{p}} \frac{p}{S}\right)
$$

where $\overline{\mathrm{S}}$ is used to take the place of $S_{0}$ in Equation 4, $p$ is the hydrostatic pressure and $\alpha_{p}$ is the pressure dependence coefficient, determined by comparing the peak yield stress in compression at constant strain rate with that in tension at the same rate.

As discussed earlier, glassy polymers exhibit rubberelasticity when the material is at a temperature $\theta$ above $\theta_{g}\left(\theta_{g}\right.$ is the polymer glass transition temperature). On the other hand, the stress-strain response of a glassy polymer shows strain hardening when it is deformed up to a large stretch at a temperature $\theta$ below $\theta_{g}$. These observations led Parks et al. [8] to model the hardening in these materials by using a statistical mechanics network model of rubber-elasticity. With the need for a new model that exhibits mathematical simplicity, that requires one test to characterize the material and that has a limited number of parameters, Arruda and Boyce developed an eight-chains model for rubberelasticity [9]. For the second barrier to inelastic flow in a glassy polymer, the constitutive form of the orientation back stress is based on an eight-chains rubber-elasticity model given by:

$$
B_{\mathrm{i}}=\frac{n k \theta}{3} \sqrt{N} L^{-1}\left(\frac{\lambda_{\text {chain }}}{\sqrt{N}}\right) \frac{\lambda_{\mathrm{i}}^{\mathrm{p}^{2}}-\frac{1}{3} I_{1}}{\lambda_{\text {chain }}}
$$

where $B_{\mathrm{i}}$ is a principal value of the deviatoric back stress tensor, the subscript $i=1,2,3$ designates one of the three principal directions, $\lambda_{i}^{p}$ are the principal values of the left Cauchy stretch tensor $\mathbf{V}^{\mathrm{p}}$, the superscript $\mathrm{p}$ designates the plastic part, $I_{1}^{\mathrm{p}}=\lambda_{1}^{\mathrm{p}^{2}}+\lambda_{2}^{\mathrm{p}^{2}}+\lambda_{3}^{\mathrm{p}^{2}}$ is the first invariant of $\mathbf{V}^{\mathrm{p}}, \lambda_{\text {chain }}=\sqrt{I_{1}^{\mathrm{p}} / 3}$ is the equivalent stretch experienced by each chain in the eight-chains network.

In the present work, we implement the model of Boyce et al. [7] along with the model of Arruda and Boyce [9] to simulate homogeneous deformation in non-filled SL5170 cured resin at room temperature deformation. We also propose a composite formulation for the elastic-viscoplastic behavior of silica-filled SL5170.

\subsection{Summary of the kinematics and kinetics}

The kinematics and kinetics of the finite deformation elastic-viscoplastic model for non-filled SL5170 cured resin are summarized below.
The elastic-plastic decomposition of the deformation gradient tensor $\mathbf{F}$ is given by:

$$
\mathbf{F}=\mathbf{F}^{\mathrm{e}} \mathbf{F}^{\mathrm{p}}
$$

where $\mathbf{F}^{\mathrm{e}}$ represents the elastic part and $\mathbf{F}^{\mathrm{p}}$ the plastic part of $\mathrm{F}$.

The velocity gradient $\mathrm{L}$ and its plastic part $\mathbf{L}^{\mathrm{p}}$ are expressed as

$$
\mathbf{L}=\dot{\mathbf{F}} \mathbf{F}^{-1}=\dot{\mathbf{F}}^{\mathrm{e}} \mathbf{F}^{\mathrm{e}^{-1}}+\mathbf{F}^{\mathrm{e}} \mathbf{L}^{\mathrm{p}} \mathbf{F}^{\mathrm{e}^{-1}}
$$

and

$$
\mathbf{L}^{\mathrm{p}}=\dot{\mathbf{F}}^{\mathrm{p}} \mathbf{F}^{\mathrm{p}-1} \cong \mathbf{D}^{\mathrm{p}}
$$

where $\mathbf{D}^{\mathrm{p}}$ is the plastic stretch of the velocity gradient and the plastic spin is assumed zero.

Polar decomposition of the plastic deformation gradient decomposes $\mathbf{F}^{\mathrm{p}}$ into a stretch and a rotation as

$$
\mathrm{F}^{\mathrm{p}}=\mathrm{V}^{\mathrm{p}} \mathrm{R}^{\mathrm{p}}
$$

The Cauchy stress tensor is

$$
\mathbf{T}=\frac{1}{J} \mathbf{C}^{\mathrm{e}}\left(\ln \mathbf{F}^{\mathrm{e}}\right)
$$

where $\mathbf{J}$ is the volume change and $\mathbf{C}^{\mathrm{e}}$ is the fourth order tensor of elastic constants.

The driving stress tensor is

$$
\mathbf{T}^{*}=\mathbf{T}-\frac{1}{J} \mathbf{F}^{\mathrm{e}} \mathbf{B} \mathbf{F}^{\mathrm{e}^{\mathrm{T}}}
$$

where the principal components of the back stress tensor $\mathbf{B}$ are given in (10).

The plastic strain rate gradient $\mathbf{D}^{\mathrm{p}}$ is given by the flow rule as

$$
\mathbf{D}^{\mathrm{p}}=\dot{\gamma}^{\mathrm{p}} \mathbf{N}
$$

where $\dot{\gamma}^{\mathrm{p}}$ is given in 4 and the resulting normalized deviatoric part of the driving stress tensor, $\mathrm{N}$, is given in terms of the deviatoric driving stress $T^{* \prime}$ as,

$$
\mathbf{N}=\frac{1}{\sqrt{2} \tau \tau} \mathbf{T}^{* \prime}
$$

\section{Experiments}

To characterize the large deformation response of silica filled and non-filled cured resin, a standard set of material tests including uniaxial tensile and compressive tests under different strain rates and different temperatures was conducted.

Uniaxial tensile tests were conducted to obtain Young's modulus and Poisson's ratio for the non-filled cured resin. The ASTM D638 [10] type I dogbone has a gauge length of $50.8 \mathrm{~mm}$, a width of $12.7 \mathrm{~mm}$ over the gage length and a thickness of $3.2 \mathrm{~mm}$. The samples were fabricated layer by layer, at $28^{\circ} \mathrm{C}$ and under the 


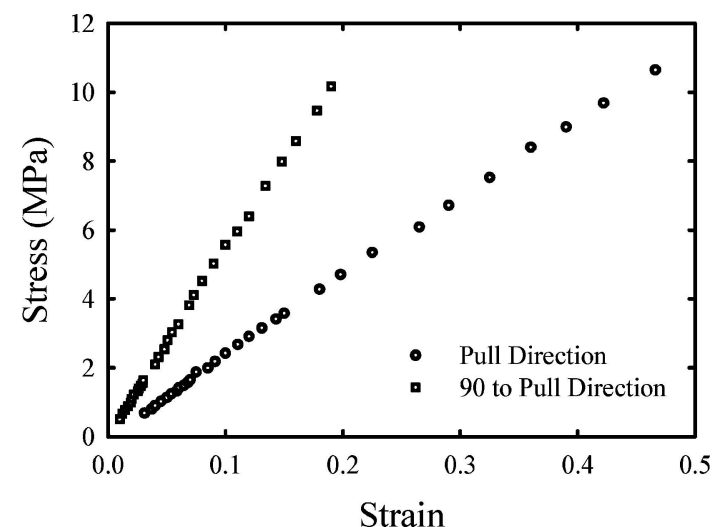

Figure 1 The initial stress-strain response of non-filled SL5170 plotted as the tensile stress versus the axial and transverse strain.

ACES standard part building style of 3D Systems Corporation. The machine is a 3D Systems SLA 250/50. The specimens were post-cured for one hour after the supports were cleaned and then exposed to laboratory atmosphere for one week. All specimens used in this work were built using the same procedure unless specified otherwise.

Micro-Measurements Group type EA-50-125TQ350 strain gauge, capable of measuring strains parallel and perpendicular to the stretch direction, was mounted to each test sample. The tensile tests were conducted on an Applied Test System (ATS) 900 Universal Testing machine. The velocity of the moving grip was $0.00508 \mathrm{~mm} / \mathrm{s}$. The initial stress-strain response is shown in Fig. 1 as the tensile stress results in axial and transverse strain. A Poisson's ratio of $v=0.43$ and a Young's modulus of $\mathrm{E}=2300 \mathrm{MPa}$ were obtained from the tension plots in Fig. 1.

Two types of cylindrical samples were used in compression tests, shown in Fig. 2. The first one (designated as type I) is an ASTM695 cylinder [11], $50.8 \mathrm{~mm}$ in height and $25.4 \mathrm{~mm}$ in diameter, used for compressive tests at small deformations and for calibrating elastic constants. The second one (designated as type II) is a small cylinder, $8 \mathrm{~mm}$ in height and $8 \mathrm{~mm}$ in diameter, designed to obtain the stress-strain response at finite strain. The layering direction is along height direction (Z-axis in Fig. 2), the compression direction is parallel to $Z$.

The type I cylinder was compressed using an INSTRON machine at room temperature under a displacement rate of $-0.0508 \mathrm{~mm} / \mathrm{s}$. The data acquisition system of the machine automatically recorded the load versus the displacement of the moving grip at a certain sampling rate. Using the initial dimensions of the sample and assuming incompressibility, the load-

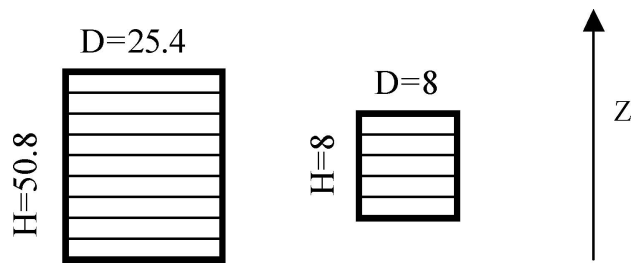

Figure 2 Specimens used for compression tests (units: $\mathrm{mm}$ ). displacement data were converted into a true stresstrue strain curve. During the compression process, thin TEFLON films were used as lubricants to reduce friction between the platens and the sample. The Young's modulus was approximately measured from the stressstrain curve to be around $2700 \mathrm{MPa}$, which is slightly different from that of the tension test. Note that SL5170 cured resin is very sensitive to the testing setup and environmental parameters including temperature and humidity. In addition, the elastic properties of SL5170 cured resin may be relevant to the deformation strain rate. Further effort is needed to investigate these factors. In our study, an average of Young's modulus from both tension and compression tests was used. The average of these values gives $E \approx(2700+2300) / 2=2500 \mathrm{MPa}$. Therefore, the Young's modulus and Poisson ratio for the non-filled SL5170 cured resin are $2500 \mathrm{MPa}$ and 0.43 , respectively.

At room temperature, type II cylinders were uniaxially compressed on the INSTRON machine under different displacement rates to investigate the effect of strain rate on the large deformation behavior. The test procedure followed for type I specimens was also used here. As shown in Fig. 3a-c, the stresses at yield were significantly different at different displacement rates. A yield strength of $75 \mathrm{MPa}$ was obtained for a displacement rate of $-0.08 \mathrm{~mm} / \mathrm{s}, 65 \mathrm{MPa}$ for a displacement rate of $-0.008 \mathrm{~mm} / \mathrm{s}$, and $55 \mathrm{MPa}$ for a displacement rate of $-0.0008 \mathrm{~mm} / \mathrm{s}$. The strain rate at small deformations is numerically equal to the ratio of the displacement rate over the initial sample height. Hence, the strain rate dependent yield stresses were concluded from these data to be: $75 \mathrm{MPa}$ at $-0.01 / \mathrm{s}$, $65 \mathrm{MPa}$ at $-0.001 / \mathrm{s}$ and $55 \mathrm{MPa}$ at $-0.0001 / \mathrm{s}$.

A type I sample was compressed uniaxially along the $Z$-axis to $36.6 \mathrm{~mm}$ in height and $31.2 \mathrm{~mm}$ in diameter: a true strain of about $32 \%$. The compressed sample was then put in an oven at $90^{\circ} \mathrm{C}$ for four hours, which is above the known glass transition temperature ranging from 60 to $80^{\circ} \mathrm{C}$ [12]. After this annealing treatment, the specimen regained its original size to within $5 \%$ dimensional difference. It is a well-known phenomenon in glassy polymers that, inelastic deformation without internal damage or reptation followed by heating above the glass transition temperature will lead to strain release.

To examine the effect of temperature on the mechanical behavior of the non-filled SL5170 cured resin, uniaxial compressive tests were performed at different temperatures $\left(25,40,60\right.$, and $\left.100^{\circ} \mathrm{C}\right)$ using type II small cylinders. The tests were conducted at a constant strain rate of $-0.001 / \mathrm{s}$ on an MTS810 servo-hydraulic testing apparatus. The samples were compressed to a predetermined strain, and then unloaded at a constant strain rate of $0.001 / \mathrm{s}$.

The stress-strain curves in Fig. 4 show the temperature dependence of non-filled SL5170 cured resin and the following conclusions can be drawn. With an increase in the temperature, the material becomes softer and the yield point drops significantly. As the temperature crosses the glass transition temperature range, there is no visible yield point on the stress-strain curve. 


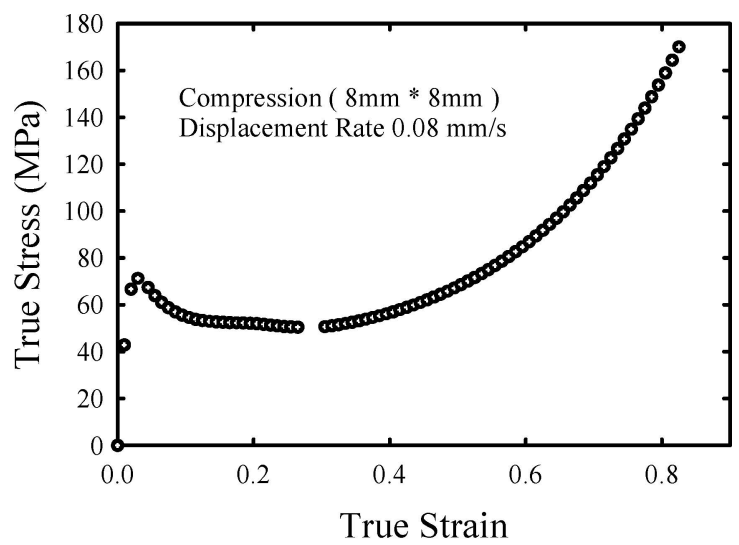

(a)

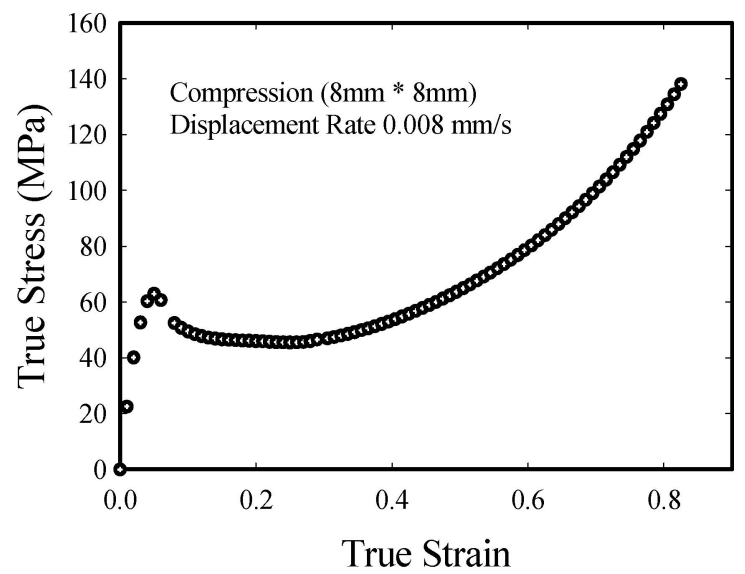

(b)

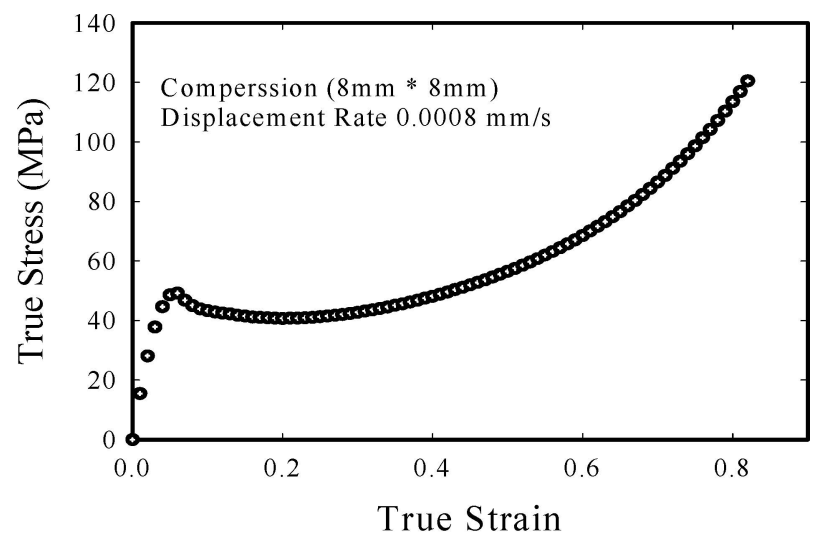

(c)

Figure 3 Stress-strain curves for non-filled SL5170 cured resin at displacement rates of (a) $-0.08 \mathrm{~mm} / \mathrm{s}$. (b) $-0.008 \mathrm{~mm} / \mathrm{s}$. (c) -0.0008 $\mathrm{mm} / \mathrm{s}$.

Actually, the material exhibits rubber-elastic behavior in that the material nearly retrieves its original dimensions after unloading eventhough it undergoes very large deformation. The specimen compressed at $100^{\circ} \mathrm{C}$ failed when deformed to about $90 \%$, which led to the discontinuation of the associated stress-strain curve.

Compression tests were also conducted for the silicafilled SL5170 resin. Powdered silica was added to SL5170 resin to form a suspension. After compounding, the suspension was introduced into a 3D System SL250(50 (equipped with a Zephyr recoater). The vat temperature was set to $28^{\circ} \mathrm{C}$, and the refined processing parameters include a Zephyr recoater waiting period (known as $Z$-wait) of $120 \mathrm{~s}$ and a critical exposure energy density (defined as Ec) of $200 \mathrm{~J} / \mathrm{m}^{2}$. The silica

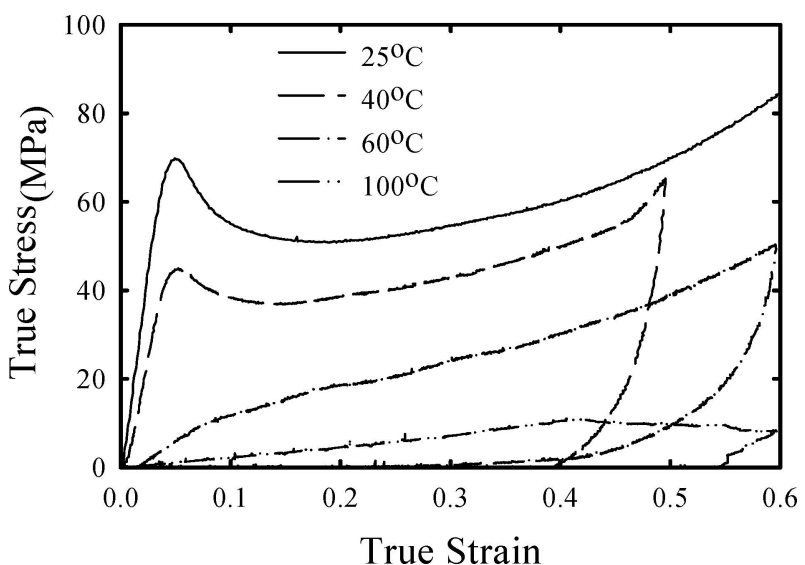

Figure 4 Compression stress-strain curves for non-filled SL5170 cured resin at different temperatures: $25,40,60$ and $100^{\circ} \mathrm{C}$, and a constant strain rate of $0.001 / \mathrm{s}$.

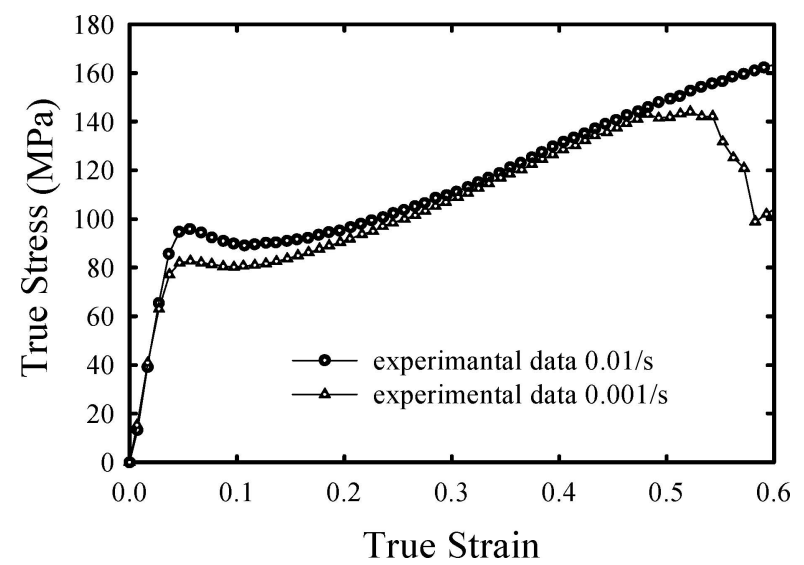

Figure 5 Compression stress-strain curves for filled SL5170 at the strain rates of $-0.01 / \mathrm{s}$ and $-0.001 / \mathrm{s}$.

used in this work had a density of $2.65 \times 10^{3} \mathrm{~kg} / \mathrm{m}^{3}$ and a tensile modulus of $73 \times 10^{3} \mathrm{MPa}$. The weight percentage of silica in the filled SL5170 was $40 \%$ and the volume fraction was 0.22 .

At room temperature and at strain rates of $-0.01 / \mathrm{s}$ and $-0.001 / \mathrm{s}$, uniaxial compression experiments were conducted using type II silica filled SL5170 cylinders. As shown in Fig. 5, the yield stresses are significantly different as the strain rate varies. A yield stress of approximate $85 \mathrm{MPa}$ was measured for a strain rate of $-0.01 / \mathrm{s}$ and $75 \mathrm{MPa}$ for a strain rate of $-0.001 / \mathrm{s}$. The stress-strain curves reflect linear elastic behavior and nonlinearity as yield is approached, strain softening after yield, followed by a steady state flow and a strain hardening.

At different temperatures $\left(25,40,60\right.$, and $\left.100^{\circ} \mathrm{C}\right)$ with the same constant strain rate of $-0.001 / \mathrm{s}$, type II cylinders made from silica filled SL5170 were uniaxially compressed and unloaded. From the stress-strain curves in Fig. 6, the filled material exhibits a temperature dependent response similar to that of the nonfilled resin, however, the filled material has a higher yield stress and a steeper strain hardening slope at each tested temperature compared to the non-filled SL5170 resin. 


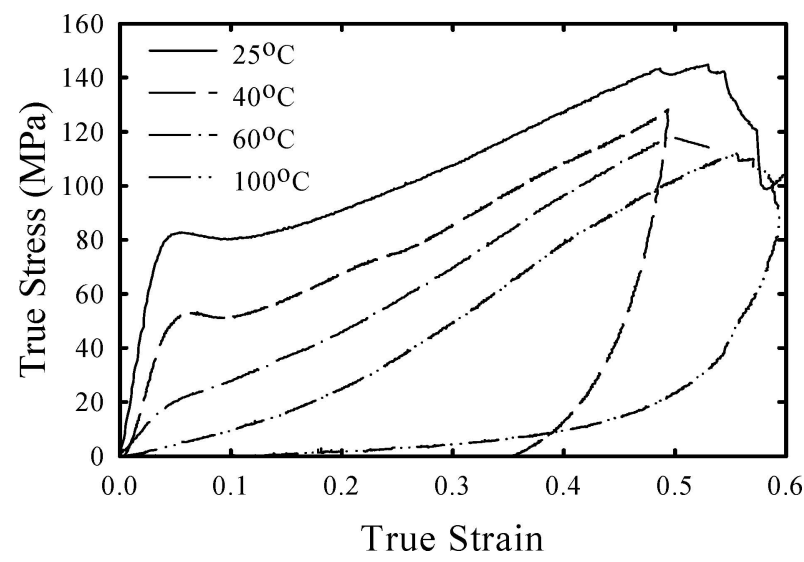

Figure 6 Compression stress-strain curves for silica filled SL5170 cured resin at different temperatures: $25,40,60$ and $100^{\circ} \mathrm{C}$ at a constant strain rate of $0.001 / \mathrm{s}$.

\section{Constitutive model for filled SL5170}

The silica used in this compound has a Poisson ratio of 0.17 [13] and Young's modulus of $73 \times 10^{3} \mathrm{MPa}$. Because silica has a high strength, the powder dispersed in the cured base behaves elastically eventhough the composite is deformed to a very large strain. In other words, the plastic deformation of the silica filled SL5170 cured resin consists of the plastic deformation of the SL5170 cured resin. It is also assumed that the silica powder is perfectly uniformly dispersed. The perfect bonding at the silica/resin interface is assumed. Based on these assumptions, the constitutive model is extended to account for the effect of the dispersed silica particles.

It is known that the velocity gradient $\mathbf{L}$ may be written as

$$
\mathbf{L}=\mathbf{D}+\mathbf{W}
$$

where $\mathbf{D}$ is the deformation rate tensor and $\mathbf{W}$ is the spin tensor. $\mathbf{D}$ can be decomposed into elastic and plastic parts

$$
\mathbf{D}=\mathbf{D}^{\mathrm{e}}+\mathbf{D}^{\mathrm{p}}
$$

Let $\mathrm{C}$ be the volume fraction of silica particles in the composite, hence we have

$$
\mathbf{D}^{\mathrm{e}}=C \mathbf{D}^{\mathrm{s}, \mathrm{e}}+(1-C) \mathbf{D}^{\mathrm{m}, \mathrm{e}}
$$

$$
\mathbf{D}^{\mathrm{p}}=C \mathbf{D}^{\mathrm{s}, \mathrm{p}}+(1-C) \mathbf{D}^{\mathrm{m}, \mathrm{p}}
$$

where $\mathbf{D}^{\mathrm{s}, \mathrm{e}}$ is the elastic portion of the strain rate in silica, $\mathbf{D}^{\mathrm{m}, \mathrm{e}}$ is the elastic portion of the strain rate in the cured SL5170 matrix, $\mathbf{D}^{\mathrm{s}, \mathrm{p}}$ is the plastic portion of the strain rate in silica, and $\mathbf{D}^{\mathrm{m}, \mathrm{p}}$ is the plastic portion of the strain rate in the matrix. The silica particles are assumed to have no plastic deformation, thus $\mathbf{D}^{\mathrm{s}, \mathrm{p}}=\mathbf{0}$. Equation 22 reduces to

$$
\mathbf{D}^{\mathrm{p}}=(1-C) \mathbf{D}^{\mathrm{m}, \mathrm{p}}
$$

Now, the Cauchy stress tensor for the composite is

$$
\mathbf{T}=\frac{1}{J} \mathbf{C}_{\mathrm{com}}^{\mathrm{e}}\left(\ln \mathbf{F}^{\mathrm{e}}\right)
$$

where $\mathbf{C}_{c o m}^{\mathrm{e}}$ is the elastic stiffness tensor of the composite material.

The stress applied to the matrix is only a portion of the total applied stress. Let the constant $b$ represent this portion, then:

$$
\mathbf{T}^{m}=b \mathbf{T}
$$

where $\mathrm{T}^{\mathrm{m}}$ is the Cauchy stress tensor for the matrix.

The driving stress tensor in the matrix is

$$
\mathbf{T}^{*}=\mathbf{T}^{m}-\frac{1}{J} \mathbf{F}^{\mathrm{e}} \mathbf{B} \mathbf{F}^{\mathrm{e} T}
$$

The plastic strain rate gradient of the cured matrix, $\mathbf{D}^{\mathrm{m}, \mathrm{p}}$, is given by the flow rule as

$$
\mathbf{D}^{\mathrm{m}, \mathrm{p}}=\dot{\gamma}^{\mathrm{m}, \mathrm{p}} \mathbf{N}
$$

with the resulting normalized deviatoric portion of the driving stress tensor, $\mathbf{N}$,

$$
\mathbf{N}=\frac{1}{\sqrt{2} \tau} \mathbf{T}^{* \prime}
$$

The elastic stiffness tensor, $\mathbf{C}_{\mathrm{com}}^{\mathrm{e}}$, is calculated using a self-consistent method proposed by Hill [14] and Budiansky [15] for the determination of the overall elastic moduli of composite materials. The composite comprises a homogenous elastic matrix (cured SL5170 matrix), in which inclusions of other elastic materials (e.g. silica particles) are firmly embedded at arbitrary concentration in such a way that the whole composite appears homogenous, on average. For an arbitrary concentration $C$ of spherical inclusions of silica, dispersed throughout the cured SL5170 matrix in a homogenous and isotropic manner on average, the composite bulk modulus, $K$, and the composite shear modulus, $G$, are calculated from the moduli of the two phases $\left(K_{\mathrm{m}}, K_{\mathrm{s}}\right.$ and $G_{\mathrm{m}}, G_{\mathrm{s}}$ ) as follows:

$$
K=K_{\mathrm{m}}+\frac{C\left(K_{\mathrm{s}}-K_{\mathrm{m}}\right)}{1+\left(K_{\mathrm{s}}-K_{\mathrm{m}}\right)(1-C) /\left(K_{\mathrm{m}}+K^{*}\right)}
$$

$$
\begin{gathered}
K^{*}=\frac{3}{2}\left(\frac{1}{G}+\frac{10}{9 K+8 G}\right)^{-1} \\
G=G_{\mathrm{m}}+\frac{C\left(G_{\mathrm{s}}-G_{\mathrm{m}}\right)}{1+\left(G_{\mathrm{s}}-G_{\mathrm{m}}\right)(1-C) /\left(G_{\mathrm{m}}+G^{*}\right)}
\end{gathered}
$$

$$
G^{*}=\frac{4}{3} G
$$


Here subscript $\mathrm{s}$ and $\mathrm{m}$ designate silica and the base matrix, respectively.

\section{Results and discussions}

The constants that must be obtained experimentally include those that model the temperature, pressure, strain rate, softening, and hardening effects. The material constants such as Young's modulus, locking stretch and coefficients for rate and pressure dependent yield are measured directly from the experimental stress-strain curves. Other parameters, such as the rubbery modulus, are selected to fit the stress-strain curves for the uniaxial compression tests.

Young's modulus and Poisson's ratio for the nonfilled SL5170 cured resin are $2500 \mathrm{MPa}$ and 0.43 at room temperature $\left(25^{\circ} \mathrm{C}\right)$, respectively. The shear modulus at this temperature is thus $\mu+900 \mathrm{MPa}$. The initial athermal shear resistance $S_{0}$ was computed from the shear modulus and Poisson's ratio at this temperature as $S_{\mathrm{o}}+122 \mathrm{MPa}$.

The locking stretch $\lambda_{\mathrm{L}}$ was determined from Fig. 3a$\mathrm{c}$ to be the stretch corresponding to the strain at which the stress increases rapidly, so $\lambda_{\mathrm{L}}=\exp \left(\left|\varepsilon_{\mathrm{L}}\right|\right)=$ $\exp (1.1)=3$.

Comparing the maximum yield stress with the minimum yield stress after softening in Fig. 3b, the ratio $S_{\mathrm{ss}}\left(S_{\mathrm{o}}\right.$ was determined to be 0.67 , thus the steady state shear resistance is $S_{\mathrm{ss}}=S_{\mathrm{o}} \times 0.67 \approx 82 \mathrm{MPa}$. The rate of the yield drop with respect to deformation is measured by $\mathrm{h}$ from Equation 8 as

$$
h \approx \frac{\Delta S}{\Delta \gamma^{\mathrm{p}}}\left(\frac{1}{1-\frac{S_{\mathrm{o}}}{S_{\mathrm{ss}}}}\right)=\frac{S_{\mathrm{ss}}}{\Delta \gamma^{\mathrm{p}}} .
$$

From Fig. 3b, $h$ was found to be $475 \mathrm{MPa}$.

The values for the constants $A$ and $\dot{\gamma}_{\mathrm{o}}$ were obtained using yield stress values from stress-strain curves at room temperature in Fig. 3a and b using Equation 4. The pressure dependence of yield was neglected in our implementation. Values for $A$ and $\dot{\gamma}_{\mathrm{o}}$ are: $\dot{\gamma}_{0}=$ $7.41 \times 10^{11} / \mathrm{s}$ and $A=118.85 \mathrm{~K} / \mathrm{MPa}$.

The silica used in this work has a tensile modulus of $73 \times 10^{3} \mathrm{MPa}$ and a Poisson ratio of 0.17 . The volume fraction of silica, $C$, is 0.22 . The constant $\mathrm{b}$, representing the portion of the total stress applied to the matrix, is estimated to be the volume fraction of the matrix, thus $b=(1-C)=0.78$.

Computer simulations of uniaxial compression deformation for non-filled SL5170 cured resin were performed at different strain rates of $-0.01 / \mathrm{s},-0.001 / \mathrm{s}$, and $-0.0001 / \mathrm{s}$ after the identification of all required material constants. The comparison between the computed stress-strain curves and the experimental results show good agreement for different strain rates (Fig. 7ac). The rate dependent yield is well predicted as well as the softening after yield, the steady state flow, and orientational hardening.

There are several factors affecting the small difference between the experimental results and the stressstrain curves predicted by the model. At first, the simulations were conducted at constant strain rates instead

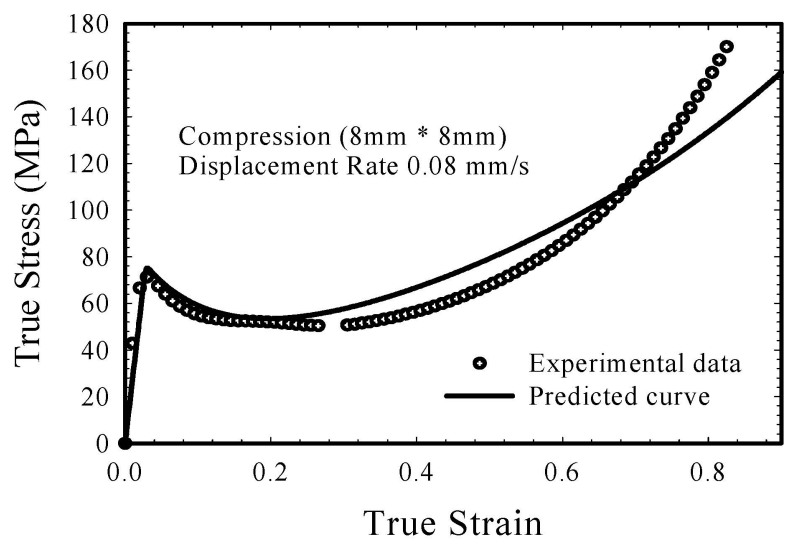

(a)

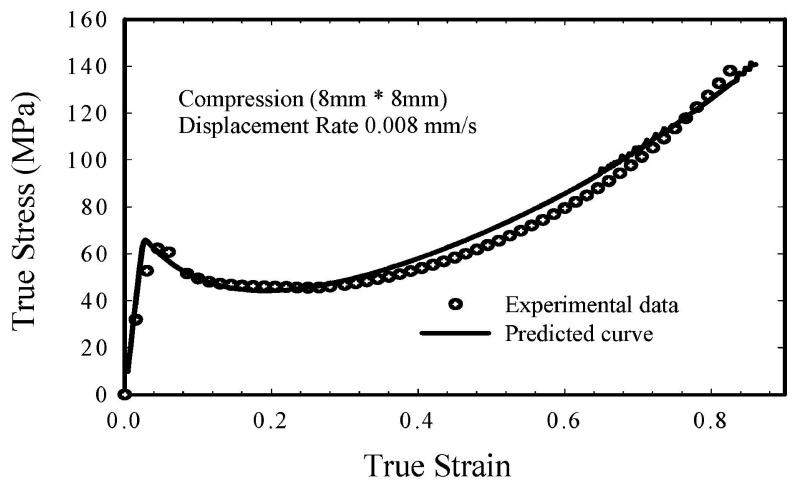

(b)

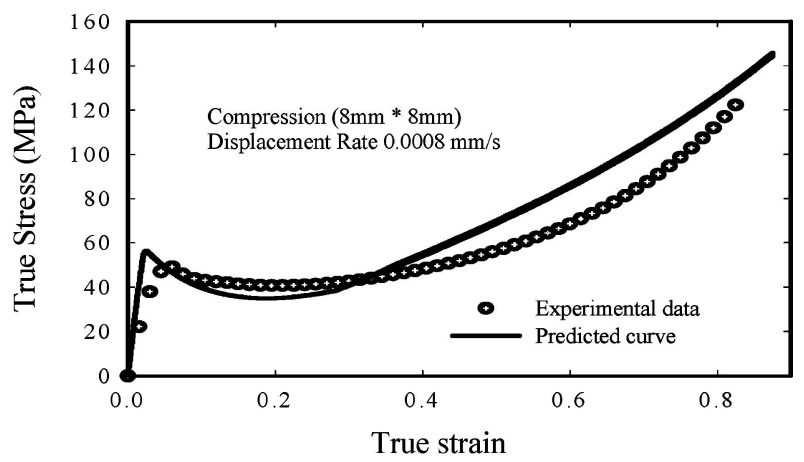

(c)

Figure 7 Compression stress-strain curves from experimental and modeling results for non-filled SL5170 at strain rate of (a) $-0.01 / \mathrm{s}$. (b) $-0.001 / \mathrm{s}$. (c) $-0.0001 / \mathrm{s}$.

of constant displacement rates, some discrepancy are therefore generated at large strains. The compression tests at room temperature for non-filled SL5170 cured resin were conducted at constant displacement rates instead of constant strain rates due to the functionality limit of the available INSTRON machines in the lab. At small strains, the ratio of the displacement rate to the initial sample height is almost identical with the strain rate. Secondly, as observed from the experimental data, the local plastic flow occurs before the peak point at the stress-strain curve. However, the developed elastic-viscoplastic model did not take this into account. Finally, due to some small discrepancy in the elastic constants, the predicted strain at which the yield stress occurs is slightly off from the experimental data.

Simulations of uniaxial compression deformation for silica-filled SL5170 cured resin were performed for strain rates of $-0.01 / \mathrm{s}$ and $-0.001 / \mathrm{s}$. The comparison between the stress-strain curves of the composite model 


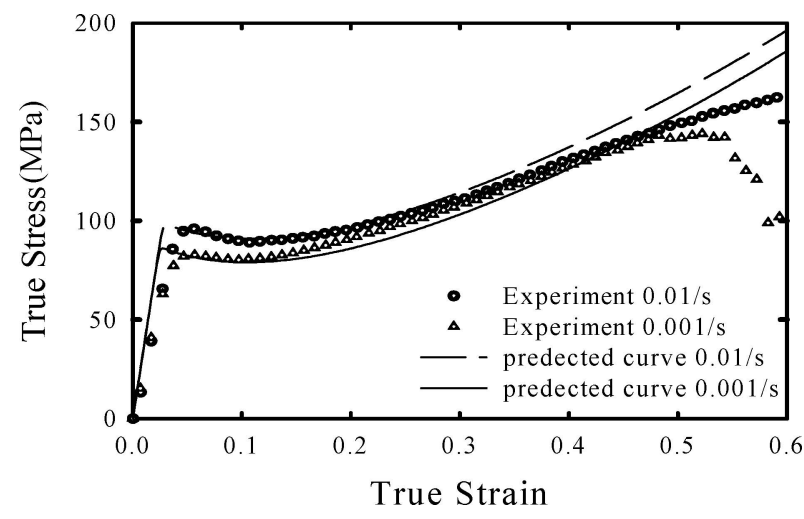

Figure 8 Compression stress-strain curves of experimental and modeling results for filled SL5170 at strain rates of $-0.01 / \mathrm{s}$ and $-0.001 / \mathrm{s}$.

and the experimental results is shown in Fig. 8 . It was observed that the simulation results of the composite model fit well the experimental data. The rate dependent yield of the composite was also well modeled. We also want to address that the composite material shows a relatively less softening and hardening than the pure cured matrix. This is due to the presence of silica particles, which changes the local microstructure response of the matrix. The silica particles restrict the polymer molecular alignment, which led to a broken sample at relatively low true strain compared to the non-filled cured matrix Fig. 8. The model composite slope of the drop with respect to plastic strain $h=350 \mathrm{MPa}$ as well as the minimum yield $S_{\mathrm{ss}}=86 \mathrm{MPa}$ are determined from the experimental data (Fig. 8).

\section{Conclusions}

In this paper, an elastic-viscoplastic mechanical model for non-filled SL5170 was used to account for rate, temperature, strain softening and hardening effect. This constitutive model was extended to account for the silica reinforcement of the silica powder filled SL5170.

After identifying material constants, the developed model successfully simulated the mechanical response of the material under homogeneous deformation such as uniaxial compression at room temperature. With the assumption that the silica particles deform elastically, the new phenomenological composite model gave a good prediction of the composite's elastic constants. The material's yield strength and the rate dependent yield, were both modeled by the composite model. The modeling results showed good agreement with the experiments.

\section{Acknowledgments}

Part of this work was supported by the ERC Program of the National Science Foundationunder Award Number EEC-9731680 with Clemson University and also by the Center for Advanced Manufacturing of Clemson University

\section{References}

1. P. F. JACOB S, Rapid Prototyping \& Manufacturing: Fundamentals of Stereolighography. (McGraw-Hill, SME, New York, 1992).

2. D. TAFT, D. OGALE, E. HUNT, S. AHZI and F. PAUL, Particulate Filled Resins for Rapid SLA Injection Tools. Solid Free Form Symposium, Austin, Texas, 1997.

3. B. WIEDEMANN, K. H. DUSEL and J. ESCHL, Investigation into the influence of material and process on part distortion. Rapid Prototyping Journal 1(1995):17-22

4. L. R. G. TRELOAR, The elasticity and related properties of rubbers. Rep. Prog. Phys. 36 (1973) 755.

5. H. M. JAMES and E. GUTH, Theory of the elastic properties of rubber. Journal of Chemical Physics 11 (1943) 455.

6. A. S. ARGON, A theory for the low-temperature plastic deformation of glassy polymers. Philosophical Magazine 28 (1973) 839.

7. M. C. BOYCE, D. M. PARKS and A. S. ARGON, Large inelastic deformation of glassy polymers. Mechanics of Materials 7 (1988) 15.

8. D. M. PARKS, A. S. ARGON and B. BAGEPALLE, Large elastic-plastic deformation of glassy polymers. MIT Program in Polymer Science and Technology Report, October, 1984.

9. E. M. ARRUDA and M. C. BOYCE, A 3-dimensional constitutive model for the large stretch behavior of rubber elastic materials. Journal of Mechanics and Physics of Solids 41 (1993) 389.

10. Annual Book of ASTM Standards, American Standard for Testing and Materials, Philadelphia, 1995.

11. Annual Book of ASTM Standards, American Standard for Testing and Materials, Philadelphia, 1992.

12. S. R. WHITE and H. T. HAHN, Process modeling of composite materials: residual stress development during cure. Part I: Model formulation. Journal of Composite Material 26 (1992) 2403.

13. P. J. HEANEY, C. T. PREWITT and V. G. GIBBS, Silica: Physical behavior, geochemistry and materials applications. Reviews in Mineralogy, Mineralogical Society of American (1994) 28.

14. R. HILL, A self-consistent mechanics of composite materials. Journal of Mechanics and Physics of Solids 13(1965) 213.

15. B. BUDIANSKY, On the elastic moduli of some heterogeneous materials. Journal of Mechanics and Physics of Solids 13(1965) 223.

Received 12 October 2004

and accepted 11 March 2005 